American Medical Journal 3 (2): 249-254, 2012

ISSN 1949-0070

(C) 2012 Science Publications

\title{
Relationship between Fetuin-A and Systemic Lupus Erythematosus as a Predictor Marker for Atherosclerosis
}

\author{
${ }^{1}$ Osama F. Mosa, ${ }^{2}$ Ibrahim H. Mohamad and ${ }^{2}$ Magdi M. Salama \\ ${ }^{1}$ Department of Health Science, Faculty of Health Science, \\ Alieth, Medical Laboratory Science Division, Umm El-Qura University, Saudi Arabia \\ ${ }^{2}$ Department of Medical Biochemistry, Faculty of Medicine, Al-Azhar University, Egypt
}

\begin{abstract}
Problem statement: Associations between serum levels of fetuin-A, C3 complement, calcium $\times$ phosphate product and calcification risk index and lipid profile in SLE patients were established. However, the mechanism of accelerated atherosclerosis accompanied with SLE remains elusive. We therefore turned to investigate the association between Fetuin-A, disease activity and accelerated atherosclerosis in patients with SLE. Approach: Serum blood samples were taken from 100 female SLE patients. All Patient samples were analyzed by ELISA for determination of Fetuin-A level. Calcium, Phosphate, C3 compelement, Lipid profile, Creatinine and urea were measured also in SLE patients compared with healthy control volunteers. Results: We found that Serum fetuin-A had been positively associated with carotid arterial stiffness, independent of known atherogenic factors in healthy subjects. Furthermore, Fetuin-A was correlated negatively with IMT, SLEDAI, CRI, CaxP product, Triglycerdies, VLDL and LDL. While it was correlated positively with C3 complement. Conclusion: Fetuin-A deficiency accompanied with increasing levels of calcium and phosphate gave an evidence that there was a key role of fetuin-A as a strong inhibitor of Cardio Vascular Calcification (CVC) by formation of a complex called (calciprotein) with calcium and phosphate in blood stream. So, Identification of biologic markers of disease activity associated with atherosclerosis may help to optimize therapy for this important manifestation of systemic autoimmune disease.
\end{abstract}

Key words: Fetuin-A, atherosclerosis, systemic lupus erythematosus, Cardio Vascular Calcification (CVC), disease activity

\section{INTRODUCTION}

SLE is an autoimmune disease in which immune system attacks the body cells and tissues, resulting in inflammation and tissue damage. SLE can affect any part of the body, but often harms the heart, joints, skin, kidney, lungs, blood vessels and central nervous system. (Manson and Rahman, 2006) SLE reflects a general defect in immune regulation that results in hyperactive $\mathrm{T}$ cells and $\mathrm{B}$ cells. The role of vascular injury in the pathogenesis of SLE is due to circulating immune complexes of autoantibodies and self antigens are deposited in the vascular wall of SLE patients and activate the complement pathway that initiate inflammatory response (Tincani et al., 2005).

The cause of SLE remains unknown. Genetic predisposition, hormonal, immunological and environmental factors trigger likely result in the disordered immune response that typifies the disease (Silva and Isenberg, 2001).
It is proposed that atherosclerosis arises as a response of the vascular wall to endothelial injury and this injury is due to endothelial apoptosis. Accelerated Atherosclerotic Vascular Disease (ASVD) is a major problem in SLE and is one of its major causes of death (Urowitz and Gladman, 2007). Coronary Artery Disease (CAD) develops in 6-9\% of SLE patients and accounts for up to $36.4 \%$ of deaths in SLE. Young and predominantly SLE are unusual group manifesting an extraordinary strong predisposition for the development of early-onset and accelerated atherosclerosis (ElMagadmi et al., 2004).

It has been suggested that a combination of traditional risk factors, including hypertension, dyslipidemia and lipid oxidation as well as nontraditional risk factors, such as autoantibodies and inflammation, may contribute to advanced vascular disease in SLE. Therefore, defining the autoimmune mechanisms that promote atherosclerosis is essential to optimize risk reduction and develop targeted

Corresponding Author: Osama F. Mosa, Department of Health Science, Faculty of Health Science, Alieth,

Medical Laboratory Science Division, Umm El-Qura University, Saudi Arabia 
therapeutics for prevention of Coronary Vascular Disease (CVD) in SLE (Harley et al., 2006).

Fetuin-A (Alpha 2-Heremans-Schimd glycoprotein, AHSG), is a glycoprotein secreted by adult liver into the peripheral circulation. This protein is commonly present in the cortical plate of immature cerebral cortex and bone marrow hemopoietic matrix (Kazama et al., 2005). Moreover it is considered to be a member of the cystatin superfamily of cysteine protease inhibitors involved in vascular pathology and bone metabolism (Fiore et al., 2007). Fetuin-A is linked to a number of cellular functions such as; brain development, bone remodeling and inhibition of soft tissue calcification by formation of a soluble colloidal microsphere of fetuin-calciumphosphate complex in blood stream and antagonist of Transforming Growth Factor- $\beta$ (TGF- $\beta$ ) (Reynolds et al., 2005; Demetriou et al., 1996).

Cardiovascular risk scores are not adequate for risk stratification in women with SLE. Measurement of coronary calcification may add information to identify asymptomatic women with lupus who might benefit from aggressive preventive measures. An elevated incidence of Cardiovascular Calcification (CVC) is observed in Hemodialysis (HD) patients. Fetuin-A is an important inhibitor of CVC. Reduced fetuin-A levels associated with inflammation and increased CVC (Cozzolino et al., 2006; Selim et al., 2006).

\section{MATERIALS AND METHODS}

Patients: 100 adult female patients (15-45 years) with systemic lupus erythematosus were admitted to the Internal Medicine Department of Medical Research Institute hospital, Alexandria University. All patients signed informed consent form for participation in the study. Confirm the diagnosis. All patients signed informed consent form for participation in the study. Presence of simultaneous infectious or inflammatory condition, malignant disease or any other condition that could significantly influence the course and outcome of disease were excluded.

Control group: 50 healthy volunteers in the control group were matched for sex and age without any chronic or acute illness. All included control subjects signed the informed consent form.

Blood sampling: Venous blood samples were collected from normal control subjects and patient groups. Immediately blood sera after draw were separated by centrifugation and stored until analysis at $-20^{\circ} \mathrm{C}$. Full history and clinical examination, with meticulous focus on the duration and activity of SLE. Were carried out for all patients. The inclusion requirements for patients' recruitment were: diagnosis of SLE according to the American College of Rheumatology (ACR) criteria and the absence of any concomitant CVC traditional risk factors (e.g., hypertension, diabetes and smoking)' Disease Activity was assessed the time of enrollment in the study using the Systemic Lupus Erythematosus Disease Activity Index (SLEDAI). Comprehensive medication histories were obtained through interviews with the patients. The use of corticosteroid therapy was categorized as current, former, or none and quantified in terms of the average daily dose over the preceding five years of the study.

\section{Biochemical parameters:}

- Serum Fetuin-A level was measured by a commercially available ELISA kit (Epitope Diagnostics, Inc) KT 800

- Serum intracellular Calcium was measured by QuantiChrom $^{\mathrm{TM}}$ Assay Kit (DICA-500)

- Serum Inorganic Phosphorous was measured by CHRONOLAB phophomolybdate UV kit (1010458)

- Lipid Profile (Total cholesterol, Triglycerides, VLDL, LDL and HDL) were measured by RANDOX UV kits on (Beckman DU-640 UV/Vis Spectrophotometer)

- Serum Creatinine was measured by QuantiChrom ${ }^{\mathrm{TM}}$ Assay Kit (DICT-500)

- Serum Urea was measured by QuantiChrom ${ }^{\mathrm{TM}}$ Assay kit (DIUR-500)

- Serum C3 complement was measured by AssayMax Human C3 complement ELISA kit (EC2101-1)

- Carotid Ultrasonography reports for the measurement of Intima-Media Thickness (IMT) and the presence of atherosclerotic plaque

- Calculation of Calcification Risk Index (CRI)

The gathered data were further used to calculate two index scores for each patient under study. The first is the 'Calcification Risk Index' (CRI), given by the formula $\mathrm{CRI}=\mathrm{Ca} \mathrm{X}$ P/Fetuin-A. (where: $\mathrm{Ca}, \mathrm{P}$ and Fetuin-A are serum levels of calcium, inorganic phosphate and Fetuin-4, respectively).

Statistical analysis were performed using: The SPSS-10 package software Student t-test was used to compare the means of the different parameters between each two groups. The correlation between the studied clinical and biochemical parameters was calculated using Pearson's correlation coefficient. 
Am. Med. J. 3 (2): 249-254, 2012

Table 1: Mean values of different biochemical studied parameters in control and SLE patient groups

\begin{tabular}{lcc}
\hline Biochemical Studied parameters & Control group & Patient group \\
\hline Fetuin-A $(\mathrm{g} / \mathrm{l})$ & $0.7 \pm 0.07$ & $0.5 \pm 0.13$ \\
Calcium $(\mathrm{mg} / \mathrm{dl})$ & $8.06 \pm 0.25$ & $8.94 \pm 0.37$ \\
Phosphate $(\mathrm{mg} / \mathrm{dl})$ & $2.97 \pm 0.20$ & $3.77 \pm 0.46$ \\
Calcium $\times$ Phosphate product $\left(\mathrm{mg}^{2} / \mathrm{dl}^{2}\right)$ & $23.92 \pm 1.91$ & $33.58 \pm 3.68$ \\
CRI & $34.49 \pm 5.44$ & $73.73 \pm 25.43$ \\
C3 Complement $(\mathrm{g} / \mathrm{l})$ & $129.70 \pm 32.20$ & $82.80 \pm 14.25$ \\
Total Cholesterol $(\mathrm{mg} / \mathrm{dl})$ & $154.30 \pm 25.65$ & $154.5 \pm 36.54$ \\
Triglycerides $(\mathrm{mg} / \mathrm{dl})$ & $73.60 \pm 18.37$ & $127.25 \pm 53.41$ \\
HDL $(\mathrm{mg} / \mathrm{dl})$ & $42.70 \pm 10.40$ & $27.70 \pm 7.14$ \\
VLDL $(\mathrm{mg} / \mathrm{dl})$ & $13.37 \pm 5.56$ & $25.45 \pm 10.68$ \\
LDL $(\mathrm{mg} / \mathrm{dl})$ & $93.70 \pm 18.25$ & $106.50 \pm 31.01$ \\
Creatinine $(\mathrm{mg} / \mathrm{dl})$ & $0.87 \pm 0.07$ & $0.99 \pm 0.13$ \\
Urea $(\mathrm{mg} / \mathrm{dl})$ & $10.17 \pm 1.73$ & $28.90 \pm 7.59$ \\
\hline
\end{tabular}

\section{RESULTS}

The basic clinical and biochemical parameters of the patients with SLE included in the study are given in Table 1. We found a significant positive correlation between age and fetuin-A ( $g / l)[r=0.209, \mathrm{p}<0.001]$. In addition, significant negative correlations were found between fetuin-A $(\mathrm{g} / \mathrm{l})$, IMT $(\mathrm{mm})$ and SLEDAI $[\mathrm{r}=-$ $0.898 \mathrm{p}<0.001, \quad \mathrm{r}=-0.685 \mathrm{p}<0.01]$ respectively. Moreover, significant negative correlation was found between fetuin-A $(\mathrm{g} / \mathrm{l})$ and CRI $[\mathrm{r}=-0.901, \mathrm{p}<0.001]$. Also, a significant negative correlation between fetuin$\mathrm{A}(\mathrm{g} / \mathrm{l})$ and Calcium x Phosphate product $\left(\mathrm{mg}^{2} / \mathrm{dL}^{2}\right)[\mathrm{r}=$ $-0.056, \mathrm{p}<0.001]$. On the other hand, significant positive correlation was observed between $\mathrm{C} 3$ complement $(\mathrm{g} / \mathrm{l})$ and fetuin-A (g/l) $[\mathrm{r}=0.780, \mathrm{p}$ $<0.001]$. Significant negative correlations were found between fetuin-A $(\mathrm{g} / \mathrm{l})$ with triglycerides $(\mathrm{mg} / \mathrm{dL}), \mathrm{LDL}$ $(\mathrm{mg} / \mathrm{dL})$ and VLDL $(\mathrm{mg} / \mathrm{dL})[\mathrm{r}=-0.291 \mathrm{p}<0.001, \mathrm{r}=-$ $0.057 \mathrm{p}<0.001$ and $\mathrm{r}=-0.291 \mathrm{p}<0.001]$ respectively.

\section{DISCUSSION}

In the present study, IMT was significantly higher in SLE group compared to control $(\mathrm{p}<0.001)$ and a significant positive correlation was observed between SLEDAI and IMT[r $=0.479, \mathrm{p}=0.033$, while a significant negative correlation was found between IMT and $\mathrm{C} 3$ complement $[\mathrm{r}=-0.790, \mathrm{p}<0.001]$. Also, there was a significant negative correlation between C3 complement and SLEDAI $[\mathrm{r}=-0.345, \mathrm{p}<0.001]$. These findings are in accordance with Roman et al. (2003) who reported that, the prevalence of atherosclerosis is significantly increased among patients with lupus and this increase is not attributable only to traditional risk factors for cardiovascular disease. The activity of the disease presented as increase of SLEDAI and lower levels of C3 complement had additional factors to atherosclerosis in SLE patients in addition to traditional risks (Roman et al., 2003).
Thomas et al. (2002) reported that many patients with SLE are likely to have asymptomatic atheromatous vascular disease. In the study of 175 women, $40 \%$ had focal plaques identified in their carotid arteries by Bmode ultrasound (Manzi et al., 1999), which are often associated with atheroma in other vascular beds, especially the coronary arteries (Frcs et al., 1994; Chambless et al., 1997). The high risk of coronary artery disease complications associated with SLE after adjustment for conventional risk factors had led to the suggestion that SLE itself is an independent cardiovascular risk factor (Urowitz and Gladman, 2000).

Fetuin-A acts as a binder of basic calciumphosphate allowing stabilization of their level in serum and preventing its precipitation. Also, fetuin-A promote phagocytosis of excess calcium so it was considered as a potent circulating inhibitor of vascular calcification (Kuznair et al., 2008). Fetuin-A is a negative acute phase reactant protein with decreased level after an inflammatory insult (Coen et al., 2006). In the current study, a significant positive correlation was found between Age and Fetuin-A $[r=0.209, p<0.01]$ in SLE patient group. These results are in agreement with Marhaug et al. (2008) who showed that fetuin-A levels in all groups of children and the adolescent were much lower than described previously in adults and there was a significant positive correlation between age and fetuin-A level (Marhaug et al., 2008). In the present study fetuin-A was significantly decrease in SLE group compared to control $(\mathrm{p}<0.001)$ and negative correlations were found between fetuin-A and IMT, SLEDAI, CRI $(r=-0.898 p<0.001, r=-0.683 p<0.001$ and $\mathrm{r}=-0.901 \mathrm{p}<0.001)$ respectively.

Ziolkowska et al. (2008) reported an inverse association between IMT and fetuin-A levels among patients with Chronic Kidney Disease (CKD). While in patients with End-Stage Renal Disease (ESRD), a positive correlation of IMT with phosphate level and age was observed (Ziolkowska et al., 2008). The mechanisms of action of fetuin-A are less understood. However, confocal microscopy and electron 
microscopy-immunogold labeling with fetuin-A suggest that the uptake of the serum protein fetuin-A by vascular smooth muscle cells is a key event in the inhibition of vesicle-mediated calcification (Proudfoot and Shanahan, 2006).

Mori et al. (2007) predicted that feutin-A level is associated with carotid arterial stiffness, independent on atherogenic factors in healthy subjects. A negative correlation was found between fetuin-A and activity of SLE disease and CRI this support previous results that; active disease and severe infections are more important causes of death in the early phase of SLE. The pathogenesis and risk factors of this premature atherosclerosis in a disease that mainly affects young women, a group usually free of atherosclerosis, are not fully understood. Coronary vasculitis is not considered to be the underlying mechanism but persistent inflammation, autoimmunity, immune complex deposition and antiphospholipid antibodies are hypothesized to cause intimal damage followed by accelerated atherosclerosis (Knockaert, 2007).

Ix et al. (2007) demonstrated an inverse correlation between mitral and aortic valve calcification and serum fetuin-A levels in a cross sectional study of 970 patients with coronary artery disease and without renal disease. While, on the other hand there is no relationship exists between fetuin-A and renal function in patients without significant renal impairment. Genetic polymorphisms affect the level of serum fetuin$\mathrm{a}$ and the risk of vascular calcification in end-stage renal disease patients. So far, this study was the first to relate valvular calcification with serum fetuin-A in patients with normal renal function. Ix et al. (2007) in the present study $\mathrm{Ca} \times \mathrm{P}$ product was increased significantly in SLE group compared with control group and there was a significant negative correlation between fetuin- $\mathrm{A}$ and $\mathrm{Ca} \times \mathrm{P}$ product. It was documented that the acceleration of aortic wall stiffening in dialysis patients is connected with medial calcification, an active cellular process controlled by calcification inducers and inhibitors. Blacher et al. (2001) also, extensive epidemiologic data demonstrated a link between calcium-phosphate metabolism derangements and cardiovascular mortality in dialysis patients. The list of calcification inducers in ESRD patients encompasses hypercalemia, hyperphosphatemia, an elevated $\mathrm{Ca} \times \mathrm{P}$ product, an increased parathyroid hormone level and excessive treatment with vitamin D (Reynolds et al., 2005; Fine et al., 1993; Spasovski, 2007).

Serum level of C3 complement was measured in the current study as indication of SLE disease activity. Serum level of C3 complement was significantly decreased in SLE patient group compared to control group and correlated negatively with SLEDAI and IMT and positively with fetuin-A in SLE patient. Complement has a big part to play in the autoimmune processes in lupus. In SLE continued complement activity due to immune complexes causes damage via the membrane attack complex, promotion of phagocyte adhesion and activation (releasing as array of inflammatory mediators and cytokines) and promotes blood clot formation (Arason et al., 2004). Deficiencies in the earlier parts of the cascade are associated with reduced ability to remove the immune complexes generated in lupus and so are often seen in lupus nephritis and vasculitis. Low concentrations of complement components due to increased catabolism are found in a majority of patients with active and sever SLE. Schur and Sandsson (1986) Along with the interpretation of our results we have to take into account that the identified relations are mainly descriptive and aim at preparing the ground for new hypothesis generation. The nature of the observed relationships as well as possible mechanisms that can direct or influence them remains to be examined.

Data obtained from the current study revealed that serum levels of triglycerides, LDL and VLDL were higher $[(\mathrm{p}<0.001, \mathrm{p}=0.241, \mathrm{p}<0.001)$ respectively] in SLE group compared to control group, while serum level of HDL $(p<0.001)$ in patients group was significantly lower compared to control. Also, significant negative correlations were observed between fetuin-A level and each of triglycerides, LDL and VLDL[r $=-0.291$ $\mathrm{p}<0.001, \mathrm{r}=-0.057 \mathrm{p}<0.001$ and $\mathrm{r}=-0.291 \mathrm{p}<0.001]$ levels in SLE patient group respectively.

These results are in consistent with Leong et al. (1994) who stated that the 'lupus pattern' is characterized by significant elevated levels of Very Low Density Lipoprotein (VLDL), Triglycerides (TG) and lower levels of High Density Lipoprotein (HDL). Although these levels were within the normal range, they were significantly higher in untreated inactive lupus patients compared to healthy controls (Leong et al., 1994).

\section{CONCLUSION}

Fetuin-A deficiency accompanied with increasing levels of calcium and phosphate gave an evidence that there was a key role of fetuin-A as a strong inhibitor of Cardio Vascular Calcification (CVC) by formation of a complex called (calciprotein) with calcium and phosphate in blood stream. So, Identification of biologic markers of disease activity associated with atherosclerosis may help to optimize therapy for this important manifestation of systemic autoimmune disease. 


\section{REFERENCES}

Arason, G.J., K. Steinsson, R. Kolla, T. Vikingsdottir and D. Ambrogio et al., 2004. Patients with systemic lupus erythematosus are deficient in complement-dependent prevention of immune precipitation. Rheumatology, 43: 783-789.

Blacher, J., A.P. Guerin, B. Pannier, S.J. Marchais and G.M. London, 2001. Arterial calcifications, arterial stiffness and cardiovascular risk in end-stage renal disease. Hypertension, 38: 938-942. PMID: 11641313

Chambless, L.E., G. Heiss, A.R. Folsom, W. Rosamond and M. Szklo et al., 1997. Association of coronary heart disease incidence with carotid arterial wall thickness and major risk factors: The Atherosclerosis Risk in Communities (ARIC) study, 1987-1993. Am. J. Epidemiol., 146: 483494.

Coen, G., P. Ballanti, A. Balducci, F. Grandi and M. Manni et al., 2006. Renal osteodystrophy: AlphaHeremans Schmid glycoprotein/fetuin-A, matrix GLA protein serum levels and bone histomorphometry. Am. J. Kidney Dis., 48: 106113. PMID: 16797392

Cozzolino, M., A. Galassi, M.L. Biondi, O. Turri and S. Papagni et al., 2006. Serum fetuin-A levels link inflammation and cardiovascular calcification in hemodialysis patients. Am. J. Nephrol., 26: 423429. PMID: 16968979

Demetriou, M., C. Binkert, B. Sukhu, H.C. Tenenbaum and J.W. Dennis, 1996. Fetuin/alpha 2-HS glycoprotein is a transforming growth factor-beta type II receptor mimic and cytokine antagonist. J. Biol. Chem., 271: 12755-12761.

El-Magadmi, M., H. Bodill, Y. Ahmad, P.N. Durrington and M. Mackness et al., 2004. Systemic lupus erythematosus: An independent risk factor for endothelial dysfunction in women. Circulation, 110: 399- 404. PMID: 15262847

Fine, A., D. Cox and B. Fontaine, 1993. Elevation of serum phosphate affects parathyroid hormone levels in only $50 \%$ of hemodialysis patients, which is unrelated to changes in serum calcium. J. Am. Soc. Nephrol., 3: 1947-1953. PMID: 8338927

Fiore, C.E., G. Celotta, G.G. Politi, L.D. Pino and Z. Castelli et al., 2007. Association of high alpha2Heremans-Schmid glycoprotein/fetuin concentration in serum and intima-media thickness in patients with atherosclerotic vascular disease and low bone mass. Atherosclerosis, 195: 110-115. PMID: 17010978
Frcs, G.G., D. Gorman, A. Nicolaides, D. Sheridan and R. Elkeles et al., 1994. Carotid intima-media thickness: Correlation with the British Regional Heart Study risk score. J. Intern. Med., 235: 431433. DOI: 10.1111/j.1365-2796.1994.tb01099.x

Harley, J.B., J.A. Kelly and K.M. Kaufman, 2006. Unraveling the genetics of systemic lupus erythematosus. Springer Semin Immunopathol., 28: 119-130. PMID: 17021721

Ix, J.H., G.M. Chertow, M.G. Shlipak, V.M. Brandenburg and M. Ketteler et al., 2007. Association of fetuin-a with mitral annular calcification and aortic stenosis among persons with coronary heart disease: Data from the heart and soul study. Circulation, 115: 2533-2539. DOI: 10.1161/CIRCULATIONAHA.106.682450

Kazama, J.J., F. Gejyo and I. Ei, 2005. The immunohistochemical localization of Alpha2Heremans-Schmid Glycoprotein/fetuin-A (AHSG). Nephrol. Dial Transplant J., 20: 851-852. PMID: 15772277

Knockaert, D.C., 2007. Cardiac involvement in systemic inflammatory diseases. Eur. Heart J., 28: 1797-1804.

Kuznair, J., T. Porazko and M. Klinger, 2008. Relationship between fetuin-A concentration, elevated levels of inflammatory markers and arterial wall stiffness in end-stage kidney disease. J. Renal Nut., 18: 83-86. PMID: 18089450

Leong, K.H., K. Et, P.H. Feng and M.L. Boey, 1994. Lipid profiles in patients with systemic lupus erythematosus. J. Rheumatol., 21: 1264-1267. PMID: 7966068

Manson, J.J. and A. Rahman, 2006. Systemic lupus erythematosus. Orphanet J. Rare Dis., 1: 1-6.

Manzi, S., F. Selzer, K. Sutton-Tyrrell, S.G. Fitzgerald and J.E. Rairie, 1999. Prevalence and risk factors of carotid plaque in women with systemic lupus erythematosus. Arthritis Rheum., 42: 51-60. PMID: 9920014

Marhaug, G., V. Shah, R. Shroff, H. Varsani and L.R. Wedderburn et al., 2008. Age-dependent inhibition of ectopic calcification: A possible role for fetuinA and osteopontin in patients with juvenile dermatomyositis with calcinosis. Rheumatology, 47: 1031-1037.

Mori, K., M. Emoto, T. Araki, H. Yokoyama and M. Teramura et al., 2007. Association of serum fetuinA with carotid arterial stiffness. Clin. Endocrinol., 66: 246-250. PMID: 17223995

Proudfoot, D. and C.M. Shanahan, 2006. Molecular mechanisms mediating vascular calcification: Role of matrix Gla protein. Nephrol. (Calrton) J., 11: 455-461. PMID: 17014561 
Reynolds, J.L., J.N. Skepper, R. McNair, T. Kasama and K. Gupta et al., 2005. Multifunctional roles for serum protein fetuin-a in inhibition of human vascular smooth muscle cell calcification. J. Am. Soc. Nephrol., 16: 2920-2930.

Roman, M.J., B.A. Shanker, A. Davis, M.D. Lockshin and L. Sammaritano et al., 2003. Prevalence and correlates of accelerated atherosclerosis in systemic lupus erythematosus. N. Engl. J. Med., 349: 23992406.

Schur, P.H. and J. Sandsson, 1986. Immunologic factors and clinical activity in systemic lupus erythematosus. N Engl. J. Med., 278: 533-538.

Selim, G., O. Stojceva-Taneva, K. Zafirovska, A. Sikole and S. Gelev et al., 2006. Inflammation predicts all-cause and cardiovascular mortality in haemodialysis patients. Prilozi, 27: 133-144. PMID: 16985487

Silva, C. and D.A. Isenberg, 2001. Aetiology and pathology of systemic lupus erythematosus. Hospital Pharmacist J., 7: 62-69.

Spasovski, G.B., 2007. Bone health and vascular calcification relationships in chronic kidney disease. Int. Urol. Nephrol., 39: 1209-1216. PMID: 17899431
Thomas, G.N., L.S. Tam, B. Tomlinson and E.K. Li, 2002. Accelerated atherosclerosis in patients with systemic lupus erythematosus: A review of the causes and possible prevention. Hon Kong. Med., J., 8: 26-32. PMID: 11861990

Tincani, A., C. Biasini-Rebaioli, R. Cattaneo and P. Riboldi, 2005. Nonorgan specific autoantibodies and heart damage. Lupus, 14: 656-659. PMID: 16218461

Urowitz, M.B. and D.D. Gladman, 2000. Accelerated atheroma in lupus-background. Lupus, 9: 161-165. DOI: 10.1191/096120300678828271

Urowitz, M.B. and D.D. Gladman, 2007. Atherosclerosis and lupus: The SLICC study. Lupus, 16: 925- 928. PMID: 18042585

Ziolkowska, H., M. Brzewski and M. RoszkowskaBlaim, 2008. Determinants of the intima-media thickness in children and adolescents with chronic kidney disease. Pediatr. Nephrol., 23: 805-811. PMID: 18228041 